Revue d'histoire de l'enfance « irrégulière »

Le Temps de l'histoire

2| 1999

Cent ans de répressions des violences à enfants

\title{
Les tribunaux face aux violences sur les enfants sous la Troisième République
}

Dominique Dessertine

\section{(2) OpenEdition \\ 12 Journals}

Édition électronique

URL : http://journals.openedition.org/rhei/35

DOI : $10.4000 /$ rhei.35

ISBN : 978-2-7535-1639-7

ISSN : $1777-540 \mathrm{X}$

Éditeur

Presses universitaires de Rennes

Édition imprimée

Date de publication : 15 novembre 1999

Pagination : 129-141

ISSN : 1287-2431

Référence électronique

Dominique Dessertine, «Les tribunaux face aux violences sur les enfants sous la Troisième

République », Revue d'histoire de l'enfance « irrégulière » [En ligne], 2 | 1999, mis en ligne le 30 juillet 2010, consulté le 04 décembre 2020. URL : http://journals.openedition.org/rhei/35 ; DOI : https:// doi.org/10.4000/rhei.35 


\section{Les tribunaux face aux violences sur les enfants}

\section{sous la Troisième République}

Dans le lent processus de polissage des mœurs à l'œuvre dans la société occidentale depuis le XVIe siècle, ${ }^{(2)}$ la législation de la Troisième République marque une étape essentielle. Désireuse de protéger les plus faibles et d'entretenir la paix sociale en une fin de siècle hantée par la criminalité, elle met en place une politique en faveur de l'enfance ${ }^{(3)}$ qui vise à la protection de la vie, mais aussi au meilleur épanouissement possible des facultés des futurs citoyens. En appelant la justice à intervenir dans la protection de l'enfance, la Troisième République rompt avec l'esprit du code civil qui privilégie la famille, protectrice "naturelle de l'enfant" et autorise le juge à intervenir contre le droit du père. La loi de 1898 "sur la répression des violences, voies de fait, actes de cruauté et attentats commis envers les enfants" s'inscrit ainsi dans tout un processus législatif, dont les principaux jalons, contemporains de l'interdiction du travail des moins de douze ans et de la scolarisation, vont de 1874 (loi qui réprime la mendicité des enfants) à 1912 (loi qui institue les tribunaux pour enfants et adolescents). Nous voudrions voir ici comment les tribunaux ont utilisé l'ensemble de l'arsenal législatif en faveur des enfants victimes de violences, de manière à bien resituer l'impact de la loi de 1898 sur la protection de l'enfance au début de la Troisième République. Nous avons donc choisi de relever tous les jugements d'actes ayant porté préjudice à des enfants, quels qu'aient été le motif de l'inculpation de leurs auteurs et le tribunal qui les a poursuivis, correctionnel ou civil. Si l'historien a bien conscience de l'importance des textes fondateurs du droit, qu'il a peut-être trop longtemps négligés, il ne peut manquer de s'interroger sur l'application des lois, qui seule permet de suivre la profondeur des changements sociaux. Notre étude des pratiques est limitée au tribu-

\section{Dominique Dessertine $^{(1)}$}

(1) Ingénieur d'études,

Centre Pierre-Léon,

CNRS UMR 5599, Uni-

versité Lumière Lyon 2.

(2) Norbert ELIAS, La

Civilisation des mours,

Paris, Calmann-Lévy,

1974, 342 p. et $L a$

Dynamique de l'Occident,

Paris, Calmann-Lévy,

1976, 324 p. (première

édition en 1939 , en allemand).

(3) Catherine ROL-

LET, La Politique à l'égard de la petite enfance sous la IIle République, Paris, PUF, INED, 1990, 2 vol, $677 \mathrm{p}$. 
(4) Dont nous avons relevé les jugements correctionnels réprimant tous les faits commis contre les enfants, quel que soit l'article du code pénal, de 1911 à 1926, en choisissant une année sur cinq, celle du recensement.

(5) Dominique DESSERTINE, La Société lyonnaise pour le Sauvetage de l'enfance. Face à l'enfance en danger, un siècle d'internat et de placement familial, Toulouse, ERES, 1990, 217 p.

(6) Dominique DESSERTINE, Bernard MARADAN, Pratiques judiciaires de l'assistance éducative (1889-1941), Centre Pierre Léon, MIRE, rapport dactylographié, 1991, 262 p.

(7) Dominique DESSERTINE, Bernard MARADAN, "La loi de 1889 et « ces orphelins qui ont le malheur d'avoir des parents "”, Autorité, responsabilité parentale et protection nal de Lyon, ${ }^{(4)}$ mais vu l'importance, dans la seconde agglomération de France, du mouvement en faveur de l'enfance dans les premières décennies de la République, ${ }^{(5)}$ nous pensons qu'elle peut offrir une solide base d'analyse des volontés réelles, sociales, profondes, de lutter contre les violences faites aux enfants par le biais de la répression judiciaire.

\section{UNE RÉPRESSION RARISSIME}

Il faut bien le constater, dès la décennie 1910, la répression en correctionnelle des délits contre les enfants ne représente qu'une vingtaine d'affaires chaque année. Ce nombre est dérisoire pour une population d'un demi million d'habitants. Il est dérisoire aussi au regard du nombre annuel de déchéances de puissance paternelle prononcées par le tribunal civil, nombre souvent deux fois plus grand et en progression constante. Il est dérisoire enfin en comparaison du nombre d'enfants traduits devant le tribunal pour enfants et adolescents, 200 en moyenne chaque année. ${ }^{(6)} \mathrm{C}$ 'est dire que, en cette fin du XIXe siècle, à un moment où l'opinion établit de plus en plus de relations entre les mauvais traitements de l'enfance et la criminalité juvénile, la répression des adolescents est dix fois plus importante que celle des actes dont sont victimes les enfants. La défense de la société prime sur la défense des plus faibles. L'exemple lyonnais est d'autant plus troublant que, dans ces premières années de la Troisième République, Lyon, plus que Paris, avait su faire preuve de zèle dans l'application de la loi de 1889 sur les enfants moralement abandonnés et avait mobilisé ses élites républicaines en faveur de la protection de ceux que Jules Simon appelait ces "orphelins qui ont le malheur d'avoir des parents". ") L'enthousiasme soulevé par la mise en œuvre de la loi de 1889 s'est éteint au bout de la décennie et le vote d'une nouvelle loi en 1898 ne l'a pas ravivé.

Le constat s'impose donc avec force. En dépit de l'émotion suscitée par les campagnes de presse dénonçant les enfants martyrs, en dépit de l'intervention du législateur, les délits contre les enfants ne sont pas réprimés. La loi de 1898 est restée lettre morte. Certains contemporains, et non des moindres, l'avaient prédit. Henry Berthélémy, le fondateur du 
Sauvetage lyonnais, un des grands défenseurs de la cause de l'enfance, savait, dès 1898, que pour punir il aurait fallu connaitre les faits et que seuls les drames les plus scandaleux émergeraient sur la place publique. ${ }^{(8)}$ Certes, le manque de mobilisation du corps social et la faiblesse des moyens de repérage peuvent être évoqués. La philanthropie républicaine s'essouffle vite dans sa volonté d'aider l'Assistance publique, en lui fournissant "des lunettes pour apercevoir où se cache le mal ", comme le dit Berthélémy. ${ }^{(9)}$ Quelques décennies de bénévolat usent les bonnes volontés, les magistrats se font plus rares au sein des œuvres, ainsi que les membres de l'enseignement primaire pourtant parmi les plus décidés dans les années 1890. ${ }^{(10)}$ D'autre part, l'Assistance publique rechigne à l'accueil de nouvelles catégories d'enfants. Les attitudes repérées par Eric Pierre $^{(11)}$ sont manifestes à Lyon aussi. Les enfants de la loi de 1898 accentuent les difficultés du placement familial pratiqué par l'Assistance publique. L'inspecteur des enfants assistés Victor Mouret, qui arrive à Lyon en 1910 (et y reste jusqu'en 1923), refuse "qu'à l'abri des récentes lois, [les juges et les philanthropes], y compris les volontaires de l'assistance privée, transforment l'assistance publique en une vaste cuvette de Ponce Pilate où chacun vient se décharger de sa responsabilité ". ${ }^{(12)}$ Il trouve iniques les lois de 1889 et 1898 car, pour lui, "soumettre les enfants naturels déjà si durement traités par les préjugés sociaux à l'influence morbide de malheureux dégénérés est une faiblesse odieuse et qu'il faut sur le champ en arrêter la propagation $"{ }^{(13)}$ Difficultés du repérage des violences sur enfants, refus des inspecteurs des enfants assistés de les recueillir au nom des dangers que ces populations d'enfants, très mal perçues, feraient courir à l'institution : on voit que les procureurs, dans une telle conjoncture, ne pouvaient guère avoir de cas à poursuivre.

Faut-il alors arrêter ici notre investigation sur les pratiques judiciaires face aux violences aux enfants? Non, bien sûr, car si les délits que les tribunaux poursuivent ne représentent qu'une infime partie de la délinquance réelle, cette délinquance apparente ${ }^{(14)}$ conserve pour nous sa signification, dans la mesure où elle signale le niveau social de l'intolérable et où la nature de cet intolérable évolue au cours des décennies. En ce sens, l'étude des jugements en correctionnelle reste riche d'enseignements, non pour analyser l'ampleur d'un phénomène, mais pour de l'enfant, Lyon,

Chronique sociale, 1992, p. $238-245$.

(8) "La répression des violences commises envers les enfants", Revue pédagogique, 1898, tome I, p. 289-303, cité par Bernard SCHNAPPER, "Le père, le procureur et l'enfant : le mythe des enfants martyrs au XIXe siècle", Voies nouvelles en histoire du droit. La justice, la famille, la répression pénale (XVIe-XXe siècles), Paris, PUF, 1991, p. 597.

(9) Henry BERTHELEMY, Rapport sur l'application des lois protectrices des enfnats moralement abandonnés, présenté au Congrès national d'assistance, Lyon, 26 juin3 juillet 1894.

(10) Dominique DESSERTINE, Bernard MARADAN, Pratiques judiciaires..., op. cit., p. 53.

(11) Voir l'article d'Eric PIERRE, dans ce même numéro.

(12) Victor MOURET, 
"De la collaboration des œuvres privées de patronage de l'enfance avec les services départementaux d'assistance”, rapport au congrès de Grenoble, 1912, p. 7.

(13) Victor MOURET, Le patronage de l'enfance coupable, étude médicolégale, Paris, Lyon, 1903, p. 70 .

(14) C. BARBERGERDAMAMME, De la criminalité apparente. Théorie et observations à partir de trois années de rapports journaliers des polices urbaines du Rhône, Lyon, 1981, 683 p. (thèse de droit dactylographiée).

(15) Jugement du

2 janvier 1911.

(16) Jugement du 14 février 1916.

(17) Michelle PERROT, "La fin des vagabonds", L'Histoire, $\mathrm{n}^{\circ} 3$ distinguer ce qui dans l'ensemble des violences faites aux enfants est le plus inacceptable.

\section{D'ABORD RÉPRIMER LA MENDICITÉ DES ENFANTS}

Plus que la violence stricto sensu, choque la mendicité à laquelle sont contraints les enfants et que la loi de 1874 (article 276 du code pénal) a permis de poursuivre. Bien que peu appliquée, elle n'en est pas moins à l'origine d'une proportion importante de jugements (plus du tiers en 1911). Ainsi, au moment même où la presse multiplie les dénonciations d'enfants martyrs et où le législateur vote de nouveaux textes, le juge se focalise sur la mendicité. Le tribunal, par exemple, en 1911, punit de deux mois d'emprisonnement Marie Philomène B., qui, en 1910, a été trouvée mendiant avec son fils de neuf ans, alors qu'il " existe à Lyon un établissement destiné à obvier à la mendicité ". L'enfant est remis à son père. ${ }^{(15)}$ Le tribunal juge aussi les chanteurs ambulants qui se font accompagner de leurs enfants, tel Louis Camille $\mathrm{H}$., chanteur ambulant rue Molière à Lyon, qui emploie sa fille Philomène, agée de moins de seize ans, " à la mendicité habituelle, soit ouvertement, soit sous l'apparence d'une profession $"{ }^{(1)}$ Les peines vont de l'amende à deux mois de prison. Rares sont les placements d'enfants décidés par le tribunal. Il faut qu'il y ait eu récidive comme dans le cas, unique dans notre sondage, d'un ménage qui vit en garni rue Moncey. Condamnés à six jours de prison en janvier 1916 pour avoir fait mendier les fillettes nées d'un premier mariage de l'épouse, les parents sont prévenus pour les mêmes faits en avril et condamnés à nouveau à la prison (15 jours cette fois), mais surtout la mère est déchue de la puissance paternelle sur ses deux enfants de neuf et douze ans, qui sont confiées à l'Assistance publique. Le mari, ayant fait appel, voit sa peine commuer à deux jours de prison.

L'activité du tribunal reflète la stratégie globale de lutte contre les vagabonds et de nettoyage de la rue, ${ }^{(17)}$ qui est à l'œuvre au XIXe siècle dans toutes les activités de protection de l'enfance et de lutte contre la criminalité juvénile. La rue est perçue comme le foyer et l'école de la 
délinquance. L'intervention de la répression est d'autant plus aisée que le délit est par nature public.

Si l'on en juge au prisme des tribunaux, à la veille de la guerre de 1914, la répression de la mendicité des enfants est tâche achevée. Alors que les conditions de vie en ville deviennent particulièrement difficiles du fait de la guerre, la répression de ce délit ne représente plus, en 1916, que le quart des jugements du tribunal (3 sur 11).

\section{POURSUIVRE LES SUPPRESSIONS DE TOUT-PETITS}

Autre type de violences réprimé par les tribunaux, les suppressions de nourrissons. Chaque année, quelques femmes ${ }^{(18)}$ sont accusées d'avoir involontairement causé la mort de leur nouveau-né (article 463 réprimant l'homicide involontaire; les infanticides, eux, sont poursuivis aux Assises). Ces femmes annoncent celles que Catherine Bonnet a décrites récemment. ${ }^{(19)}$ Elles font un déni de grossesse, soit psychologique, soit social. Franceline G., 22 ans, domestique, a accouché le 7 juillet 1911. Le jugement porte "qu'elle n'avait rien préparé en vue de son accouchement, [qu'] elle avait soigneusement caché sa grossesse à tout le monde, sans appeler à son aide, [qu'] elle a elle-même coupé le cordon ombilical, [qu'] elle prétend qu'aussitôt le cordon coupé elle a été prise d'une syncope et qu'elle a dîu laisser son enfant sans soins sous les couvertures du lit, mais rien ne vient démontrer la réalité de cette version et même des traces de strangulation existeraient mais qu'en laissant de côté cette dernière particularité, il n'en reste pas moins certain que la fille G. a laissé son enfant nouveau-né sans aucun soin et qu'elle a ainsi causé sa mort par négligence ». Elle est condamnée à un an de prison. ${ }^{(20)}$ Dans d'autres cas, quand il n'est pas démontré que l'enfant ait vécu (article 345), la peine est de un mois à cinq ans. Ainsi, Anne Marguerite P. est condamnée à trois ans de prison pour avoir supprimé, en novembre 1908 et janvier 1910, deux enfants ${ }^{(21)}$ dont il n'est pas démontré qu'ils aient vécu.

Il n'y a guère d'évolution dans le nombre de ces délits. Ils semblent témoigner d'une frange particulièrement stable de grossesses niées, forme ultime de conduite malthusienne. ${ }^{(2)}$ En revanche, les poursuites

(18) 2 en 1911, 1 en 1916, 2 en 1921, 2 en 1926.

(19) Catherine BON-

NET, Geste d'amour.

L'accouchement sous $X$,

Paris, O. Jacob, 1990.

(20) Jugement du

23 novembre 1911.

(21) Jugement du

16 novembre 1911.

(22) Que l'on retrouve dans l'entre-deux-guerres dans un nouveau type de jugements quand le tribunal correctionnel se voit remis les jugements pour avortement, décriminalisé par la loi de 1920. Les cas restent peu nombreux (2 en 1926). Les femmes accusées 
d'avoir avorté sont punies de mois de prison avec sursis. Parfois, quand il s'agit d'une très jeune fille, une mesure de placement est prise à son encontre.

(23) Cas de MarieLouise G. qui y dépose son enfant de cinq mois (jugement du

11 décembre 1911).

(24) Les actes que nous avons relevés concernent des individus soit renvoyés faute de preuves (1 en 1911, 1 en 1921), soit condamnés au titre de l'article 312 du code pénal revu par la loi de 1898.

Notons que, sur les 5 affaires de 1926, 3 seulement mentionnent "article 312 et loi de 1898”, une l'article 312 sans précision, une enfin les articles 309 et 311 .

(25) Audience des époux M., 37 et 38 ans, prévenus de mauvais traitements sur leur enfant de moins de douze ans, le 15 mars 1911; en abandon disparaissent peu à peu. Quelques mères (une en 1911, trois en 1916) sont jugées pour avoir abandonné, "dans un lieu non solitaire " (palier du premier étage d'un immeuble ${ }^{(23)}$ ), un enfant dont l'âge n'est pas toujours mentionné, mais qui est déclaré « hors d'état de se protéger en raison de son état physique " (article 350 et 352 du code pénal). Elles sont condamnées à des peines variant de quinze jours de prison avec sursis à deux mois ferme. L'abandon dans ces conditions est condamnable mais peu condamné, ces femmes, célibataires ou veuves, attirant la compassion plus que la condamnation. Ce grand fléau du XIXe siècle qu'est l'abandon des enfants est en voie de disparition; il devient exceptionnel. Les poursuites disparaissent même au lendemain de la Grande Guerre. Une meilleure insertion économique des femmes, un moins grand opprobre contre les mères célibataires et surtout une meilleure prise en charge des enfants et de leur mère peuvent expliquer la fin de ce type de répression.

\section{UNE SENSIBILITÉ GRANDISSANTE AUX COUPS}

Les actes de violences prévenus ${ }^{(24)}$ restent extrêmement rares (3 en 1911, 2 en 1916), mais sont de plus en plus poursuivis (9 en 1926). En effet ils représentent moins de $20 \%$ des poursuites en 1911, mais plus du tiers en 1926. Il n'en reste pas moins que les juges hésitent à réprimer les quelques individus qui leur sont déférés. Ils concluent par un renvoi : « les débats de l'audience, jugent-ils, n’ont pas suffisamment fait la preuve du délit reproché aux prévenus ${ }^{{ }^{(25)}}$ Ils demandent une expertise médicale qui semble ne pas déboucher. ${ }^{(26)}$ Surtout ils se contentent de sanctionner les mères et ne le font que pour des cas extrêmes où il est indispensable de retirer l'enfant pour lui sauver la vie. Les peines restent légères. En 1911 une cultivatrice qui battait sa fillette de quatre ans, la privait d'aliments et de soins, au point " de compromettre gravement sa santé», faits attestés par un médecin et un commissaire de police, est condamnée à deux mois de prison avec sursis ${ }^{(27)}$ (au titre de l'article 312 du code pénal, modifié par la loi de 1898). Une seconde condamnation, après récidive, lui vaut six jours de prison ferme et s'assortit d'un retrait définitif de ses droits sur la petite Jeanne, 
confiée à l'Assistance publique. ${ }^{(28)}$ Une autre mère est condamnée pour des faits semblables à trois mois de prison; son fils est également confié à l'Assistance publique. ${ }^{(29)}$ Notons que ces femmes étaient mariées, mais que le père de l'enfant maltraité n'est pas inquiété.

Dans les années 1920, la violence "semble" de plus en plus souvent repérée et jugée, même s'il faut toujours se rappeler que ne se distinguent ici que des tendances sur une répression quasiment nulle. En outre les accusations se diversifient. Les mères ne sont plus seules à être observées et dénoncées. Un premier père est incriminé, en 1921, pour avoir frappé son fils à coups de pied. Il est condamné à huit jours de prison. ${ }^{(30)}$ Des couples sont aussi accusés, ${ }^{(31)}$ ce qui est nouveau, tout comme le fait que les parents ne sont plus seuls en cause. Une nourrice est également condamnée à deux mois de prison, des témoins ayant rapporté les cris quotidiens d'un garçon de dix ans qu'elle violentait et avait enfermé à peine vêtu dans une chambre sans feu en plein hiver ${ }^{(32)}$. Pour la première fois est poursuivi un surveillant de maison d'enfants, âgé de quarante-neuf ans, auteur de coups et de gifles sur plusieurs enfants. Il est condamné à $50 \mathrm{~F}$ d'amende. ${ }^{(3)}$

Si les coups ont pu être considérés comme des châtiments corporels s'intégrant dans un système éducatif, ${ }^{(3)}$ au XXe siècle les juges rompent avec cette vision des faits. Les gifles elles-mêmes ne sont plus tolérées audelà d'une certaine vigueur. Désormais on poursuit les pères qui les distribuent systématiquement. Les Italiens sont facilement repérés (une affaire sur trois en 1926). Ils acceptent mal leur condamnation et font appel. Ce décalage de perception culturelle s'observe aussi entre citadins et ruraux, ces derniers apparaissant particulièrement brutaux aux juges, surtout quand ils élèvent les coups au rang de principe éducatif. Le 10 janvier 1926, Claude B., cultivateur à Saint-Germain-sur-l'Arbresle coupe les cheveux de son petit Jean, âgé de deux ans et demi. La scène tourne mal. L'enfant a le malheur de ne pas rester immobile et le père le bat si fort que le lendemain un certificat médical peut attester que « les traces de doigts sont encore apparentes » sur sa joue gauche. Le juge dénie au père le droit de "soutenir qu'il a agi dans la limite de ses droits de correction »; il estime " que le jeune âge de l'enfant ne pouvait, même s'il avait commis une faute, être exercé avec audience des époux G., le 25 juillet 1921.

(26) Cas de Jean-

Marie J., employé au PLM, qui pourrait être incriminé au titre de l'article 312 pour coups sur son fils Marcel, de moins de quinze ans (7 novembre 1916).

(27) Jugement du 21 juin 1911.

(28) Jugement du 18 mars 1912.

(29) Jugement du 29 mars 1916.

(30) Jugement du 19 décembre 1921.

(31) Les époux L. sont condamnés à $50 \mathrm{~F}$ d'amende, le 19 décembre 1921.

(32) Jugement du 23 mai 1921.

(33) Jugement du 1er juillet 1926.

(34) Voir Bernard SCHNAPPER, op. cit., et celui de Pascale QUINCY-LEFEBVRE dans ce même numéro. 
(35) Jugement du 7 juillet 1926, arrêt en appel du 17 novembre 1926.

(36) Georges VIGARELLO, Histoire du viol (XVIe-XXe siècles), Paris, Seuil, 1998, p. 188.

(37) Dont un coiffeur de 30 ans, installé route de Vienne, condamné à 10 mois de prison avec sursis (jugement du 18 décembre 1911).

(38) L'un des auteurs est en outre condamné pour excitation de mineurs à la débauche. une pareille violence, qu'il a aggravé son attitude par les propos qu'il a tenu à cet enfant en lui disant: "Je suis maître de mon enfant, $j$ 'ai droit de vie et de mort sur lui" ». Il est condamné à un mois de prison, que son appel voit confirmer, mais avec sursis. ${ }^{(3)}$ Le droit du père reste très fort.

La rareté des cas s'accompagne d'une très grande légèreté des condamnations par rapport au texte de la loi. Les femmes sont incriminées plus que les hommes. En outre, rares sont les enfants soustraits à la violence des parents : trois sont placés à l'Assistance publique, un quatrième est confié à une tierce personne, c'est-à-dire moins du tiers de la totalité des enfants victimes que nous avons repérés. L'article 5 de la loi, qui prévoit la possibilité pour le tribunal correctionnel de statuer définitivement sur la garde de l'enfant, n'est que peu appliqué.

Aussi peu utilisée qu'elle ait été, la loi révèle un élargissement du regard social, qui s'écarte du milieu familial pour repérer quelques professionnels de l'enfance, nourrices et éducateurs. Mais n'est-ce pas aussi parce que la publicité des faits est à travers eux mieux avérée? La famille, elle, reste un lieu clos. La loi de 1898 reste vraiment fantomatique.

\section{LES VIOLENCES SEXUELLES}

L'inceste, le viol, crimes sexuels, échappent à notre investigation puisqu'ils sont de la compétence des Assises. Mais on sait que la violence sexuelle, à la fin du XIXe siècle, relève de plus en plus fréquemment du correctionnel, du fait même du juge d'instruction. ${ }^{\left({ }^{(3)}\right.}$

Seuls sont réprimés en correctionnelle les outrages publics à la pudeur des enfants. Il faut, comme l'indique le chef d'inculpation, que le délit ait été commis en présence de témoins pour qu'il tombe sous le coup de la loi. C'est dire que nos jugements ne révèlent qu'une part infime des abus dont pouvaient être victimes les enfants. Il n'en reste pas moins que, plus encore que les coups, les outrages publics à la pudeur (article $330 \mathrm{du}$ code pénal) sont de plus en plus souvent poursuivis ( 2 cas en $1911,{ }^{(37)} 3$ en $1916,{ }^{(38)} 8$ en 1921, 4 en 1926), ainsi que les attentats à la pudeur, moins nombreux. Ces violences-là ne sont pas pardonnées aux étrangers. Or, 
pendant la guerre, les déplacements de populations multiplient les risques. En 1916, sont mis en accusation un Polonais ${ }^{(39)}$ et un Serbe. ${ }^{(40)}$ Les accusés sont des exhibitionnistes qui attirent des enfants, généralement en groupe, parfois à proximité des écoles ${ }^{(4)}$ ou sur les bords déserts des quais de Saône, ${ }^{(42)}$ et leur offrent pastilles et chocolats. ${ }^{(4)}$ Certains se font caresser ou passent leurs mains sous la jupe des fillettes, d'autres ont des gestes indécents sur des petits garçons. ${ }^{(4)}$ Les peines vont de la prison avec sursis à des peines de prison ferme en cas de récidive. ${ }^{(4)}$ Mais les circonstances atténuantes sont souvent accordées aux condamnés.

Quant à l'attentat à la pudeur (article 331 du code pénal), il ne relève du tribunal correctionnel que s'il est commis sans violences. ${ }^{\left({ }^{(6)}\right.}$ Un seul cas apparait dans notre sondage, en 1916, et montre que le tribunal fait preuve d'indulgence devant ce qui doit lui apparaittre comme un jeu sexuel traditionnel. Le jeune garçon de seize ans, prévenu d'attentat à la pudeur sans violences sur deux fillettes de moins de treize ans, est remis à son père sans autre condamnation que le paiement des frais de justice. ${ }^{(4)}$

Les jugements pour excitations de mineures à la débauche, qui condamnent ceux qui prostituent des mineures (3 en 1911, 1 en $1916,{ }^{(48)}$ 2 en 1921, 7 en 1926) sont également peu nombreux, mais ils témoignent d'une répression grandissante. Sont poursuivis quelques personnages douteux, hommes et femmes, tenant cafés et garnis tant dans le centre de Lyon (rue Lanterne) que dans des petites villes ouvrières $\left(\right.$ Givors ${ }^{(49)}$ ), qui savent parfois pertinemment que leurs "bonnes" sont mineures. Les peines vont de vingt jours à deux ans de prison, et à cinq ans d'interdiction de séjour quand les coups sont venus s'ajouter à la prostitution. Un proxénète de moins de dix-huit ans est poursuivi pour de tels faits et reconnu comme ayant agi avec discernement; il est condamné à six mois de prison ${ }^{(50)}$ et cinq ans d'interdiction de séjour. Les débits de boissons où se sont déroulés les faits sont fermés.

Après le vote de la loi de 1921 réprimant le vagabondage des mineurs, le tribunal intensifie - relativement! - ses poursuites et, en 1926, apparaissent des jugements qui laissent percevoir une démarche plus affirmée de lutte contre l'abus sexuel sur les enfants. Un débitant de boissons est ainsi convaincu d'avoir pratiqué à diverses reprises des attou-
(39) Jugement du 1er juillet 1916.

(40) Mécanicien à Monplaisir, qui se masturbait en présence de jeunes enfants (jugement du 6 juillet 1916).

(41) Jugement du 18 janvier 1921.

(42) Jugement du 14 décembre 1911.

(43) Jugement du 1er juillet 1916.

(44) Jugement du 8 juillet 1926.

(45) Jugement du 14 décembre 1911.

(46) Le 28 juillet 1926, le tribunal se déclare incompétent : l'attentat à la pudeur a été commis avec violence.

(47) Jugement du 10 janvier 1916.

(48) Plus celui de la double inculpation évoquée plus haut.

(49) Jugements des 31 mai 1911 et 12 juillet 1921.

(50) Jugement du 30 novembre 1911. 
(51) Jugement du 4 janvier 1926.

(52) Jugement du 15 février 1926.

(53) Jugement du 27 août 1926. Albert P., qui est accusé d'avoir favorisé sa débauche, est condamné à dix mois de prison et cinq ans d'interdiction de séjour, condamnation dont le réhabilite l'arrêt du 7 mai 1946. chements obscènes sur des fillettes de onze et douze ans. Les enfants sont considérées comme consentantes; il est condamné à trois mois de prison. Est également condamné à quinze mois de prison, Félix B., cinquantecinq ans, marchand de primeurs, qui attirait chez lui des jeunes filles de treize, quinze et seize ans et avait des relations avec l'une d'entre elles en présence des autres ${ }^{(51)}$ Apparaît aussi la première affaire de mère coupable, celle d'une veuve de quarante et un ans qui livrait sa fille de douze ans à ses clients avant de coucher à son tour avec eux en présence de l'enfant. La mère est déchue et sa fille Anne, convaincue par ailleurs de vagabondage, est confiée au Sauvetage de l'enfance jusqu'à sa majorité. ${ }^{(52)}$ Pour la première fois en matière de violence sexuelle, les jugements prennent en compte le sort de l'adolescente victime. La jeune Renée G., qui se livrait à la débauche, est acquittée pour avoir agi sans discernement et placée à la Ruche, "maison de retenue" des diaconesses de Reuilly à Paris. ${ }^{\left({ }^{(3)}\right)}$

Globalement, dans les premières décennies du XXe siècle, s'affirme une volonté de mieux poursuivre au nom de la loi de 1898, mais surtout d'intervenir dans le domaine sexuel. Il y a un déplacement majeur des préoccupations judiciaires et, au-delà, sociales. Mais on n'aurait pas la pleine image du rôle joué par les tribunaux dans la répression des violences faites aux enfants, si on se contentait de prendre en compte l'activité des tribunaux correctionnels. Autant que celle de 1898, la loi de 1889, qui s'applique au civil, a joué un rôle dans la répression des violences commises sur les enfants.

\section{LE RÔLE MÉCONNU DE LA LOI DE 1889}

La loi sur la déchéance songeait plus à mettre en place des procédés de prévention de la délinquance, à veiller sur la sécurité ou la moralité des enfants qu'à préserver les victimes de coups. Toutefois elle a été la première à offrir une intervention judiciaire contre les mauvais traitements aux enfants. Rappelons que l'article 2 de cette loi prévoit que seront déchus "les père et mère qui par leur ivrognerie habituelle, leur inconduite notoire et scandaleuse ou par de mauvais traitements compromettent soit la 
santé, soit la sécurité soit la moralité de leurs enfants ". L'étude systématique de son application dans la région lyonnaise que nous avons menée il y a quelques années ${ }^{(54)}$ a mis en relief une orientation des tribunaux que n'avait pas prévue le législateur.

- les tribunaux ont poursuivi plus de femmes, mères célibataires ou veuves, que de pères.

- ils les ont jugées plus sur une vie sexuelle débridée, un abandon moral que pour des coups sur leurs enfants.

- ils leur ont retiré leur enfant plus pour des faits de négligence et défaut de soins que pour des violences systématiques.

Toutefois, à Lyon au moins, la loi de 1889 a permis de protéger des violences autant d'enfants que la loi de $1898 .{ }^{(5)}$ Au cours des deux premières décennies, leur nombre ne cesse de s'accroittre et, à la veille de la guerre de 1914, ils représentent $40 \%$ des enfants dont les parents sont déchus. ${ }^{(56)}$ Peut-on dire que, dans un premier temps, l'application de la loi de 1898 a écarté les enfants maltraités de la protection de la loi de 1889? Les données sont trop faibles statistiquement pour permettre de conclure. En revanche, on peut souligner qu'en correctionnelle les juges ont tendance à ne pas prononcer de déchéance. Jean-Louis R., tonnelier à Villeurbanne, "alcoolique qui ne travaille pas régulièrement ", est condamné en correctionnelle, en 1905, à huit jours de prison avec sursis pour s'être livré à des violences et à des voies de fait sur ses trois enfants, dont l'âge s'échelonne de 8 ans à 1 an. Dans le jugement ${ }^{(5)}$ qui prononce la déchéance trois mois plus tard, il est porté que le "tribunal aurait dû alors prononcer la déchéance ». Les deux démarches semblent bien distinctes, héritages des pratiques antérieures à la loi de 1898, ainsi que l'illustre, entre autres, le cas de la petite Joséphine G. de six ans, fille d'une mère, célibataire, qui la « rouait de coups, la frappait fréquemment avec une canne [...], lui faisait tenir les bras en croix et la battait cruellement lorsque épuisée de fatigue l'enfant laissait retomber ses bras [...], qu'ily a lieu de croire que le cartilage brisé du nez de l'enfant l'a été par un des nombreux coups reçus par elle». La mère est condamnée le 18 avril 1896 à six mois de prison. Sa déchéance est prononcée lors de sa sortie de prison. ${ }^{(58)}$

Les violences incriminées sont particulièrement lourdes : en 1901, Pauline M. était frappée "continuellement " par son père qui la "privait

(54) Dominique DES-

SERTINE, Bernard

MARADAN,

"Pratiques... ", rapport

cité note 6 ; et

Dominique DESSERTI-

NE, Bernard MARA-

DAN, "La loi de 1889 et

"ces orphelins..."”,

article cité note 7 .

(55) Nous comparons

ici les données des

quatre années 1911 à

1926 pour lesquelles

nous disposons des juge-

ments relatifs aux deux

lois : en 1911, 6 loi de

1889 contre 3 loi 1898 ;

en 1921, 4 contre 5 ; en

1926, 6 contre 5 .

(56) 1 en 1891, 8 en

1896, 5 en 1901, 10 en

1906, 6 en 1911.

(57) Jugement du

tribunal civil du 8 jan-

vier 1906.

(58) Jugement du tri-

bunal civil du

22 octobre 1896. 
(59) Jugement du tribunal civil du 21 mars 1901.

(60) Jugement du tribunal civil du 15 novembre 1906.

(61) Voir l'article de Jean-Jacques YVOREL dans ce même numéro. de nourriture ". ${ }^{\left({ }^{99)}\right)}$ Elles sont souvent la conséquence de l'alcoolisme : dans ses crises de delirium un père va jusqu'à jongler avec ses enfants, de trois et cinq ans, "qu'il jette en l'air au risque de les estropier». ${ }^{\left({ }^{(6)}\right)}$ Elles peuvent ne concerner qu'un seul enfant de la famille, comme la petite Henriette P., trois ans et demi en 1891, dont le père est condamné une première fois à trois mois de prison par la Cour d'appel et est déchu le 17 décembre suivant. Il a quatre enfants, et seule Henriette est martyrisée : "sa naissance paraît avoir été une grande déception dans le mariage, le père l'accueillant ainsi : Tu peux crever si tu veux».

La déchéance qui retire l'enfant à ses parents est une mesure autrement plus radicale que l'intervention ponctuelle d'une condamnation pour coups.

\section{CONCLUSION}

Peu mobilisée par la répression des mauvais traitements et violences à enfants, la justice se limite à réprimer quelques comportements publics. Elle hésite à intervenir au sein de la famille. Certes, nous n'avons pas pris en compte, dans cet article, les jugements d'assises, car nous tenions à éliminer tous les crimes et à repérer essentiellement les "violences ordinaires" que la loi devait réprimer. ${ }^{\left({ }^{(1)}\right)} \mathrm{Il}$ faut donc constater cette timidité des pratiques judiciaires. La justice a besoin de journalistes pour alerter l'opinion, de témoins pour dénoncer les faits et d'associations pour repérer et recueillir les petites victimes. La mobilisation reste largement insuffisante au-delà des émotions populaires entretenues par la presse à grand tirage, contemporaine des premières lois de protection de l'enfance. Toutefois, même extrêmement rares, les jugements des tribunaux révèlent une double évolution : d'une part, la disparition de certains comportements comme l'abandon des enfants ou la mendicité et, d'autre part, au lendemain de la première guerre mondiale, une sensibilité croissante à la violence, en particulier sexuelle, commise sur des enfants ou des adolescents.

Ainsi, l'analyse des jugements révèle combien socialement le texte qui dit la loi est fondamental, puisqu'il permet de dénoncer l'insupportable. 
Mais elle révèle aussi combien la répression ne saurait suffire à mettre un terme aux comportements dont sont victimes les enfants. Condition nécessaire mais non suffisante à la lutte contre la violence, la loi de 1898 peut être lue comme une des premières étapes engagées par la société occidentale pour protéger l'enfant des coups, mais non comme un apport révolutionnaire : condamner n'est pas résoudre. Peut-être les juges contemporains en avaient-ils obscurément conscience? Le traitement judiciaire ne saurait être le seul à faire face aux problèmes causés par les violences sur les enfants. C'est ce que l'échec relatif de l'application des textes répressifs a fait découvrir aux intervenants sociaux. 